\title{
Schizophrenia, Bipolar Disorder, and Alzheimer's Disease are not Causal Factors of Bone Mineral Density: A Mendelian Randomization Analysis
}

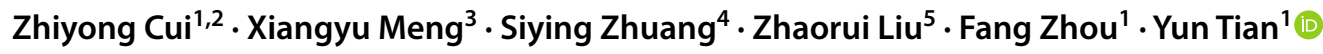

Received: 5 September 2019 / Accepted: 15 October 2019 / Published online: 2 November 2019

(c) The Author(s) 2019

\begin{abstract}
Until recently, it remains unclear whether schizophrenia, bipolar disorder (BD), and Alzheimer's disease (AD) is associated with bone mineral density (BMD). We aimed to investigate the causal effects of schizophrenia, BD and AD on BMD with Mendelian randomization (MR) analysis. Single-nucleotide polymorphisms (SNPs) strongly associated with these three neuropsychiatric diseases as instrumental variables were selected from genome-wide association studies in the MR Base database. We analyzed the effects of these SNPs on the femoral neck BMD (FN-BMD), lumbar spine BMD (LS-BMD) and forearm BMD (FA-BMD), and evaluated the heterogeneities and pleiotropy of these genetic variants. We also evaluated the potential confounding factors in the association between these three neuropsychiatric diseases and the BMD level. It was found that none of these genetic variants were significantly associated with BMD or confounding factors. Using these genetic variants, we did not find statistically significant causal effects of per unit increase in the log-odds of having schizophrenia, BD or AD with FN-BMD, LS-BMD and FA-BMD changes (e.g. schizophrenia and FN-BMD, MR-Egger OR 0.9673, 95\% CI 0.8382 to $1.1163, p=0.6519)$. The MR results also revealed that directional pleiotropy was unlikely to bias the causality (e.g., schizophrenia and FN-BMD, intercept $=0.0023, p=0.6887$ ), and no evidence of heterogeneity was found between the genetic variants (e.g., schizophrenia and FN-BMD, MR-Egger $Q=46.1502, I^{2}=0.0899, p=0.3047$ ). Our MR study did not support causal effects of increased risk of schizophrenia, BD and AD status with BMD level.
\end{abstract}

Keywords Bone mineral density $\cdot$ Schizophrenia $\cdot$ Bipolar disorder $\cdot$ Alzheimer’s disease $\cdot$ Mendelian randomization

Zhiyong Cui, Xiangyu Meng, and Siying Zhuang contributed equally to this work.

Electronic supplementary material The online version of this article (https://doi.org/10.1007/s00223-019-00625-x) contains supplementary material, which is available to authorized users.

Yun Tian

tiany@bjmu.edu.cn

1 Department of Orthopedic Surgery, Peking University Third Hospital, No 49 Huayuan Road, Haidian District, Beijing, China

2 Peking University Health Science Center, Beijing, China

3 Department of Urology, Zhongnan Hospital of Wuhan University, Wuhan, Hubei, China

4 Wuhan University School of Medicine, Wuhan, Hubei, China

5 Peking University Sixth Hospital, Beijing, China

\section{Introduction}

Osteoporosis is the most common bone disease, and it is characterized by low-bone mass, deterioration and disruption of bone structure [1]. The measurement of bone mineral density (BMD) has been proved to be an effective method for diagnosing osteoporosis and assessing the risk of fragility fracture [2]. Although osteoporosis is an important and common public health problem, the mechanisms and risk factors underlying osteoporosis and BMD are still poorly understood.

Schizophrenia, bipolar disorder (BD), and Alzheimer's disease (AD) are three common neuropsychiatric diseases. Schizophrenia is a complex and severe psychiatric disorder that affects patients' actions, perceptions, emotions, and cognitive functions [3]. Bipolar disorder (BD) is a chronic and recurrent psychiatric disorder that leads to cognitive and functional impairment and increased mortality [4]. Alzheimer's disease $(\mathrm{AD})$ is a neurodegenerative disease that is 
associated with advanced age. AD is also the primary cause of dementia in the elderly people [5]. Observational studies have suggested that these three neuropsychiatric diseases are associated with lower BMD and an increased risk of fracture [6-8]. However, because of the confounding factors between these three disorders and BMD, it is unclear whether they are etiologically relevant to BMD or are correlates of other unmeasured confounding factors. Well-conducted randomized controlled trials are the gold standard for inferring causality, but randomization to the neuropsychiatric diseases' condition is not possible. Therefore, we used Mendelian randomization (MR), which is a widely used causal inference method that uses genetic variants as instrumental variables (IVs) to assess the causal relationship between the three neuropsychiatric diseases and BMD $[9,10]$. The genetic variants in MR are available with the progress of genome-wide association studies (GWAS) and high-throughput genomic technologies.

In this study, we used single-nucleotide polymorphisms (SNPs) strongly associated with schizophrenia, BD and AD as IVs. We performed a two-sample MR using the effect of IVs on the exposures (schizophrenia, BD and $\mathrm{AD}$ ) and outcomes (BMD) from two independent samples. We analyzed the summary-level data to obtain quantitative estimates of the causal effects of schizophrenia, BD and AD on the femoral neck BMD (FN-BMD), lumbar spine BMD (LS-BMD) and forearm BMD (FA-BMD) to investigate the potential role of three neuropsychiatric diseases on FN-BMD, LSBMD and FA-BMD.

\section{Materials and Methods}

We used SNPs as IVs for all exposures (schizophrenia, BD and AD). We selected SNPs with a genome-wide association $(p<5 \mathrm{E}-08)$ and independent inheritance $\left(r^{2}<0.01\right)$ without any linkage disequilibrium (LD) from summary statistic data from GWAS in the MR Base database (http:// www.mrbase.org/). The European samples from the 1000 genomes project were adopted to estimate LD between chosen SNPs. When target SNPs were not available in the outcome (FN-BMD, LS-BMD and FA-BMD) study, we used proxy SNPs that were in high LD $\left(r^{2}>0.8\right)$ with the SNPs of interest. SNPs associated with schizophrenia were derived from a multi-stage GWAS study performed by Psychiatric Genomics Consortium (PGC) Schizophrenia Working Group, which consisted of 36,989 cases and 113,075 controls of European and Asian ancestry [11]. SNPs associated with BD were the results of 7481 cases and 9250 controls in an European ancestry GWAS conducted by PGC Bipolar Disorder Working Group [12]. For AD, we used the publicly available summary statistics data sets of GWAS metaanalyses in individuals of European descent (17,008 cases and 37,154 controls) from the International Genomics of Alzheimer's Project (IGAP) [13]. The summary-level data of GWAS for the impact of the exposures-associated SNPs on FN-BMD, LS-BMD and FA-BMD were obtained from the Genetic Factors for Osteoporosis (GEFOS) Consortium, including 508,253 osteoporotic fractures patients of European ancestry and 53,236 European ancestry individuals in the general population [14]. The detailed characteristics of GWAS associated with exposures (schizophrenia, BD and $\mathrm{AD})$ and outcomes (BMD) are showed in Table S1 in the Supplementary Material.

We applied the principles of two-sample MR to assess the role of exposures (schizophrenia, BD and AD) in the susceptibility of outcomes (BMD). First, we assessed the independent association of SNPs with schizophrenia, BD and $\mathrm{AD}$, and selected SNPs that were strongly associated $(p<5 \mathrm{E}-08)$ and independent inheritance $\left(r^{2}<0.01\right)$ without any LD with the three neuropsychiatric diseases. Then, we obtained the effect estimates for the selected SNPs on FN-BMD, LS-BMD and FA-BMD from the GEFOS release in 2015. Third, we combined the summary statistics (beta coefficients and standard errors) to estimate the causal associations between these three neuropsychiatric diseases and BMD using two-sample MR analysis. We used three methods for MR analysis: inverse variance weighting (IVW), weighted median (WM) and MR-Egger regression. The IVW method uses a meta-analysis approach to combine the Wald ratios of the causal effects of each SNP to yield a consistent causal estimate. The SNPs included had to satisfy the assumptions of an instrumental variable. The WM estimate provides a consistent estimate of the causal effect if at least $50 \%$ of the weight contributing to the analysis comes from effective IVs. MR-Egger regression, based on the assumption that the pleiotropic associations are independent, performs a weighted linear regression of the outcome coefficients on the exposure coefficients. However, MR-Egger estimates may be inaccurate and can be strongly influenced by outlying genetic variants. The WM estimate has been confirmed to have distinct superiorities over MR-Egger for its improved power of causal effect detection and lower type I error. We also used the IVW, WM and maximum likelihood methods to evaluate the heterogeneities between SNPs. The heterogeneities were quantified by Cochran $Q$ statistics and $I^{2}$ statistics. To guarantee that the MR estimates are not influenced by the inclusion of proxy SNPs, we implemented a sensitivity analysis by removing a different SNP in each iteration when performing the MR. Moreover, we used the MR-Steiger method to test causal directions between the exposures and outcomes.

We performed MR-Egger regression to explore and adjust for pleiotropy, which refers to the phenomenon in which a single genetic locus affects multiple phenotypes. The intercept represents the average pleiotropic effect across 
the genetic variants. We performed a systematic search and identified some BMD risk factors to evaluate the potential confounding factors in the association between exposures and outcomes. The BMD risk factors included obesity indices (body mass index (BMI), waist circumference, hip circumference and waist-to-hip circumference ratio), glycemic traits (type 2 diabetes, fasting glucose and 2-h glucose), systolic and diastolic blood pressure, smoking behavior, alcohol intake behavior and inflammatory bowel diseases (IBD). Summary statistics for obesity indices (BMI, waist circumference, hip circumference and waist-to-hip circumference ratio) were extracted from studies performed by the Genetic Investigation of ANthropometric Traits (GIANT) consortium [15-17], glycemic traits (type 2 diabetes, fasting glucose and 2-h glucose) from GWAS meta-analyses by DIAbetes Genetics Replication And Meta-analysis (DIAGRAM) Consortium [18] and Meta-Analyses of Glucose and Insulin-related traits Consortium (MAGIC) [19, 20], systolic and diastolic blood pressure from the International Consortium of Blood Pressure (ICBP) [21], smoking behavior from a GWAS meta-analysis conducted by the Tobacco and Genetics Consortium (TGC) [22], alcohol consumption behavior from samples in the UK Biobank (UKB) [23] and IBD from International Inflammatory Bowel Disease Genetics Consortium (IIBDGC) [24]. The detailed characteristics of studies associated with confounding factors are showed in Table 1. We assessed the potential associations between SNPs that were extracted for the MR analysis and those confounding factors. Given that BMD outcomes were derived from the same summary-level data of GWAS, associations of the SNPs with three BMD outcomes were considered statistically significant at a Bonferroni-corrected $p<0.05 /(3 \times N)$, and associations of the SNPs with the confounding factors were considered statistically significant at a Bonferroni-corrected $p<0.05 / N$, with $N$ representing the number of SNPs in each exposure trait. All statistical tests were two-sided, and the results of the MR analyses were considered statistically significant at a Bonferroni-corrected $p<0.0167$ (e.g., $0.05 / 3$ outcomes). All MR analyses were performed using the "TwoSampleMR" package for R language, version 3.6.0 (R Foundation for Statistical Computing, Vienna, Austria).

\section{Results}

\section{Causality Between Schizophrenia and BMD}

Based on independent and LD analyses, we selected fortynine independent SNPs $\left(p<5 \mathrm{E}-08, r^{2}<0.01\right)$ associated with schizophrenia in European and Asian ancestry [11] to analyze the causality with FN-BMD, LS-BMD and FA-BMD in the MR Base database. After removing five
SNPs (rs11139497, rs11191419, rs12325245, rs215411, rs2851447) that were palindromic with intermediate allele frequencies (palindromic SNPs referred to the SNPs with $\mathrm{A} / \mathrm{T}$ or $\mathrm{G} / \mathrm{C}$ alleles and "intermediate allele frequencies" referred to $0.01<$ allele frequency $<0.30$ ), forty-four SNPs remained to perform the MR analysis for each BMD trait. None of the forty-four SNPs were significantly associated with BMD outcomes $(p<0.0004)$ (e.g., 0.05/132), as shown in Table 1. Our results did not suggest causal associations of per unit increase in the log-odds of having schizophrenia with FN-BMD, LS-BMD and FA-BMD changes because none of genetic variants were significantly associated with BMD at the Bonferroni-corrected significance threshold $(p<0.0167)$ based on IVW, WM and MR-Egger regression methods (For FN-BMD, MR-Egger OR 0.9673, $95 \%$ CI 0.8382 to $1.1163, p=0.6519$; IVW OR 0.9954, 95\% CI 0.9624 to $1.0296, p=0.7907$; WM OR 0.9870 , $95 \%$ CI 0.9424 to $1.0338, p=0.5804$. For LS-BMD, MREgger OR $0.9916,95 \%$ CI 0.8419 to $1.1678, p=0.9195$; IVW OR $1.0040,95 \%$ CI 0.9667 to $1.0427, p=0.8375$; WM OR $0.9753,95 \%$ CI 0.9231 to $1.0304, p=0.3725$. For FA-BMD, MR-Egger OR 1.2108, 95\% CI 0.8976 to $1.6334, p=0.2173$; IVW OR $0.9944,95 \%$ CI 0.9294 to $1.0641, p=0.8718$; WM OR $1.0257,95 \%$ CI 0.9319 to 1.1291, $p=0.6037$.) (Table 2 and Fig. 1). We conducted the MR-Egger regression to assess the pleiotropy, and the results revealed that directional pleiotropy was unlikely to bias the causality with FN-BMD (intercept $=0.0023$, $p=0.6887)$, LS-BMD (intercept $=0.0010, p=0.8789)$ and FA-BMD (intercept $=-0.0156, p=0.1929)$ (Table 3). The associations between these genetic variants and confounding factors were analyzed. None of the forty-four genetic variants were significantly associated with the confounding factors mentioned above at the Bonferroni-corrected significance threshold $(p<0.05 / 44=0.0011)$ (Tables S2-S7). Cochran's $Q$ value and the $I^{2}$ value indicated no evidence of heterogeneity between IV estimates with IVW, MR-Egger and maximum likelihood methods (For FN-BMD, MR-Egger $Q=46.1502, I^{2}=0.0899, p=0.3047$; IVW $Q=46.3290, I^{2}=0.0719, p=0.3366$; Maximum likelihood $Q=46.3274, I^{2}=0.0718, p=0.3367$. For LS-BMD, MR-Egger $Q=42.4492, I^{2}=0.0106, p=0.4516$; IVW $Q=42.4729, I^{2}<0.0001, p=0.4940$; Maximum likelihood $Q=42.4725, I^{2}<0.0001, p=0.4940$. For FA-BMD, MR-Egger $Q=30.5206, I^{2}<0.0001, p=0.9057$; IVW $Q=32.2717, I^{2}<0.0001, p=0.8842$; Maximum likelihood $Q=32.2714, I^{2}<0.0001, p=0.8843$.) (Table 3). We evaluated the sensitivity analysis by a "leave-one-out" analysis and found that no single SNP was driving the MR estimates (see Fig. S1 in the Supplementary Material). The MR-Steiger results supported the causal direction between the exposures and outcomes (Table 1). 


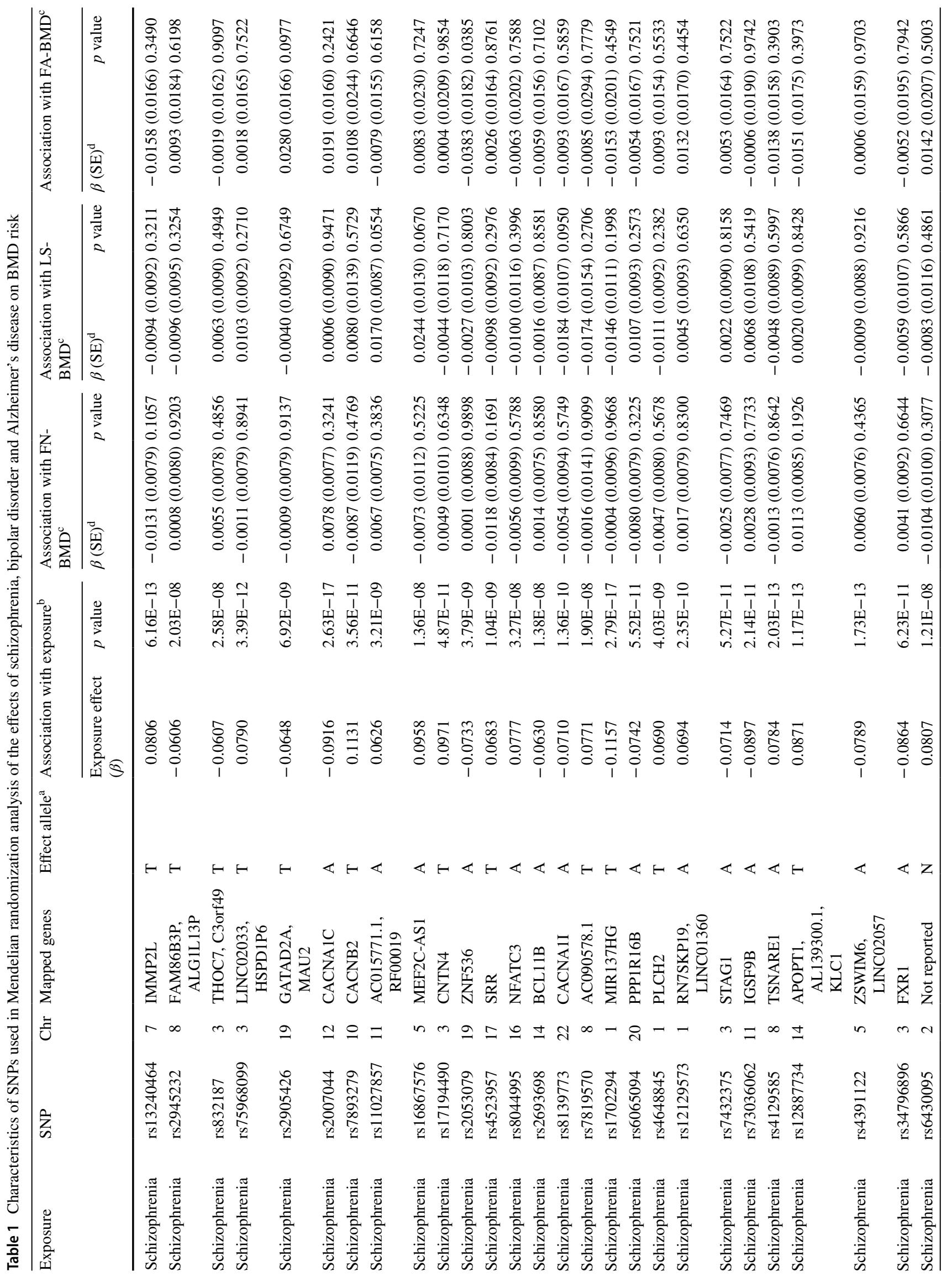




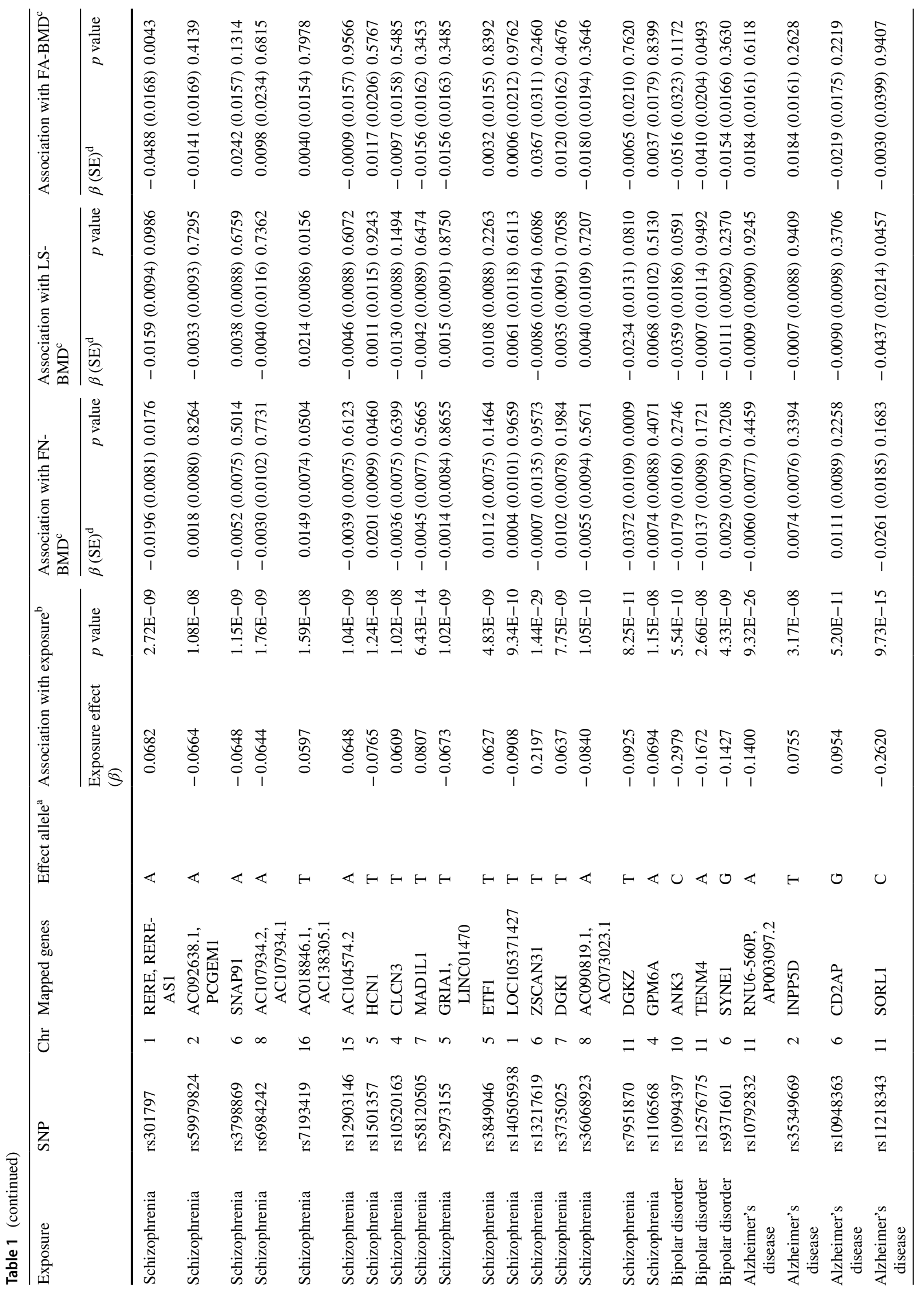




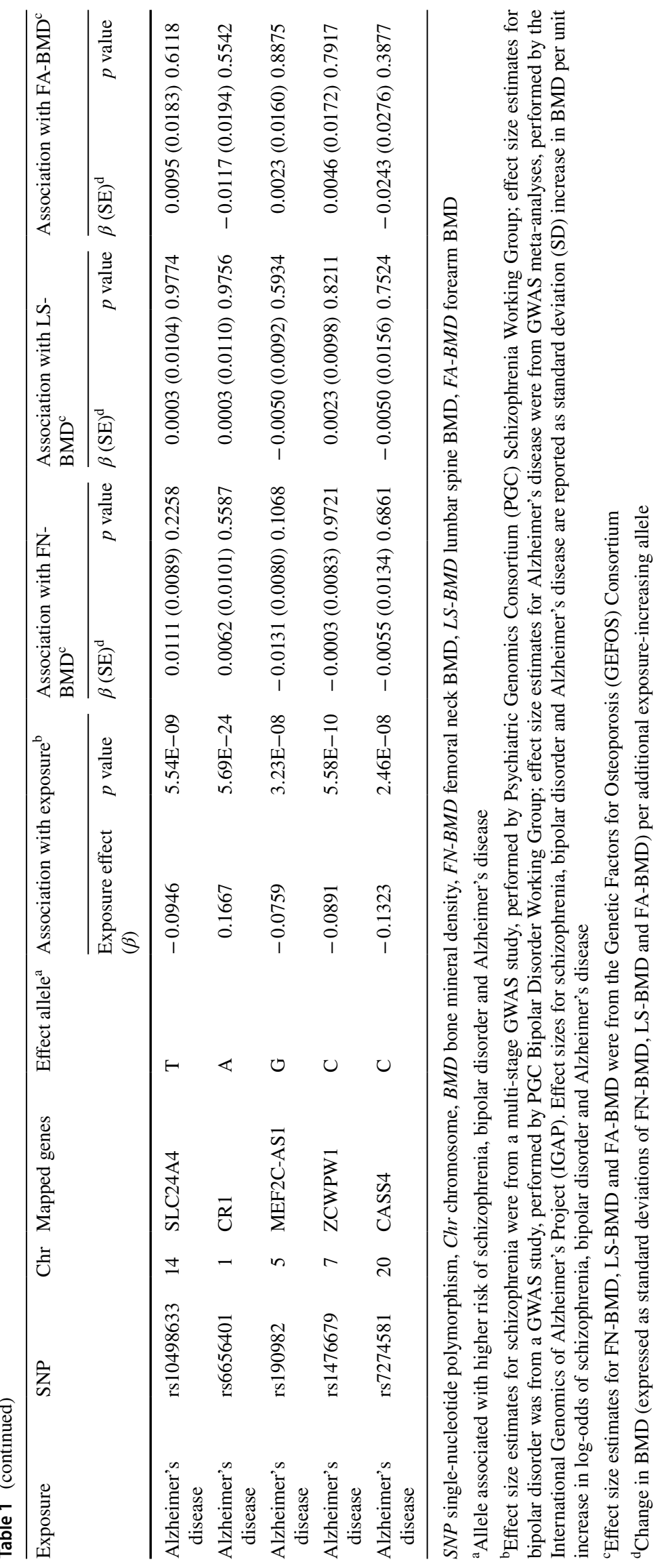




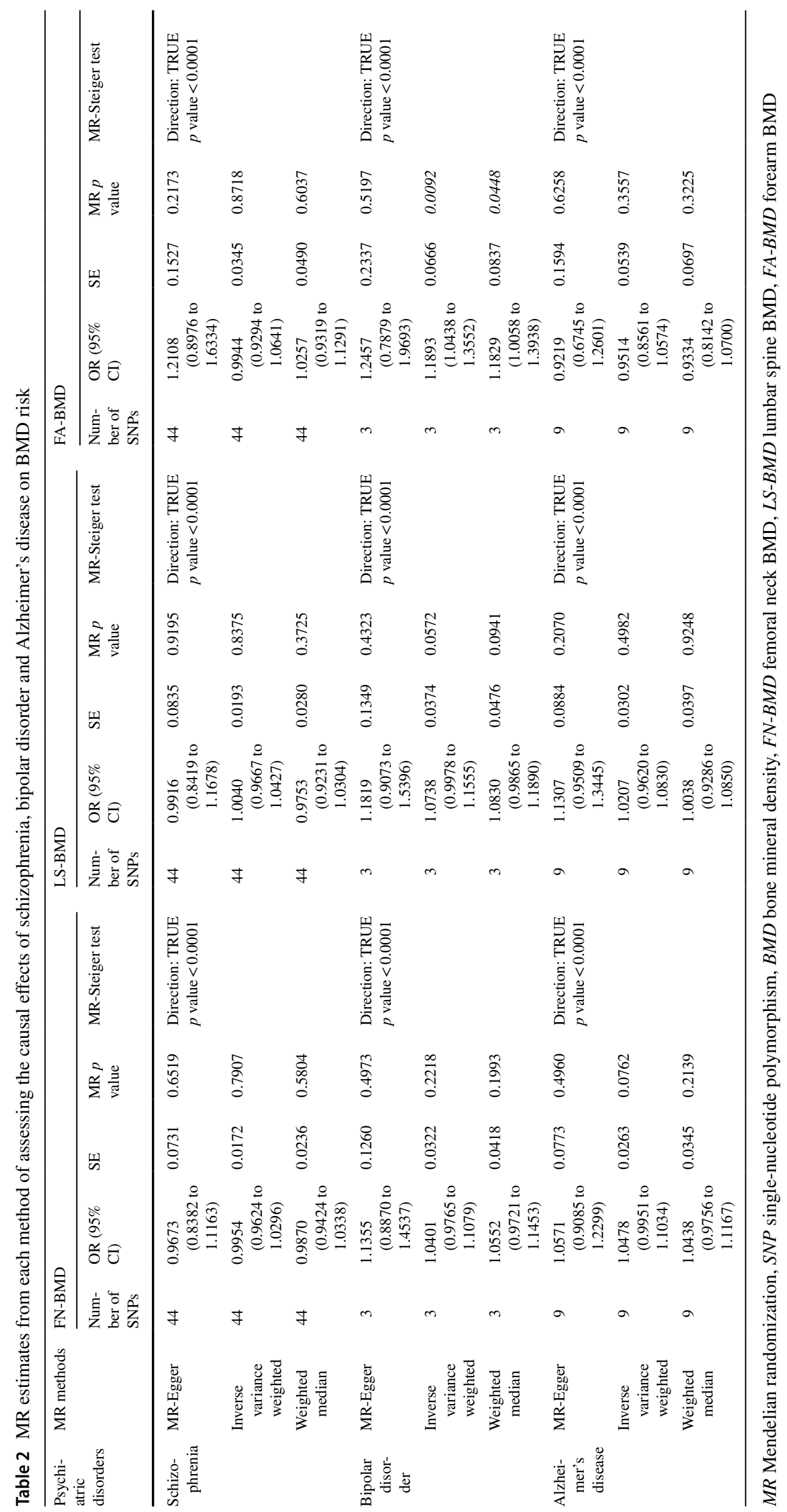


Fig. 1 Scatter plots of genetic associations with schizophrenia against BMD using different MR methods. a Schizophrenia and FN-BMD results; $\mathbf{b}$ Schizophrenia and LS-BMD results; and c Schizophrenia and FA-BMD results. The slopes of each line represent the causal association for each method. The blue line represents the inverse variance weighted estimate, the green line represents the weighted median estimate, and the dark blue line represents the MR-Egger estimate (a)
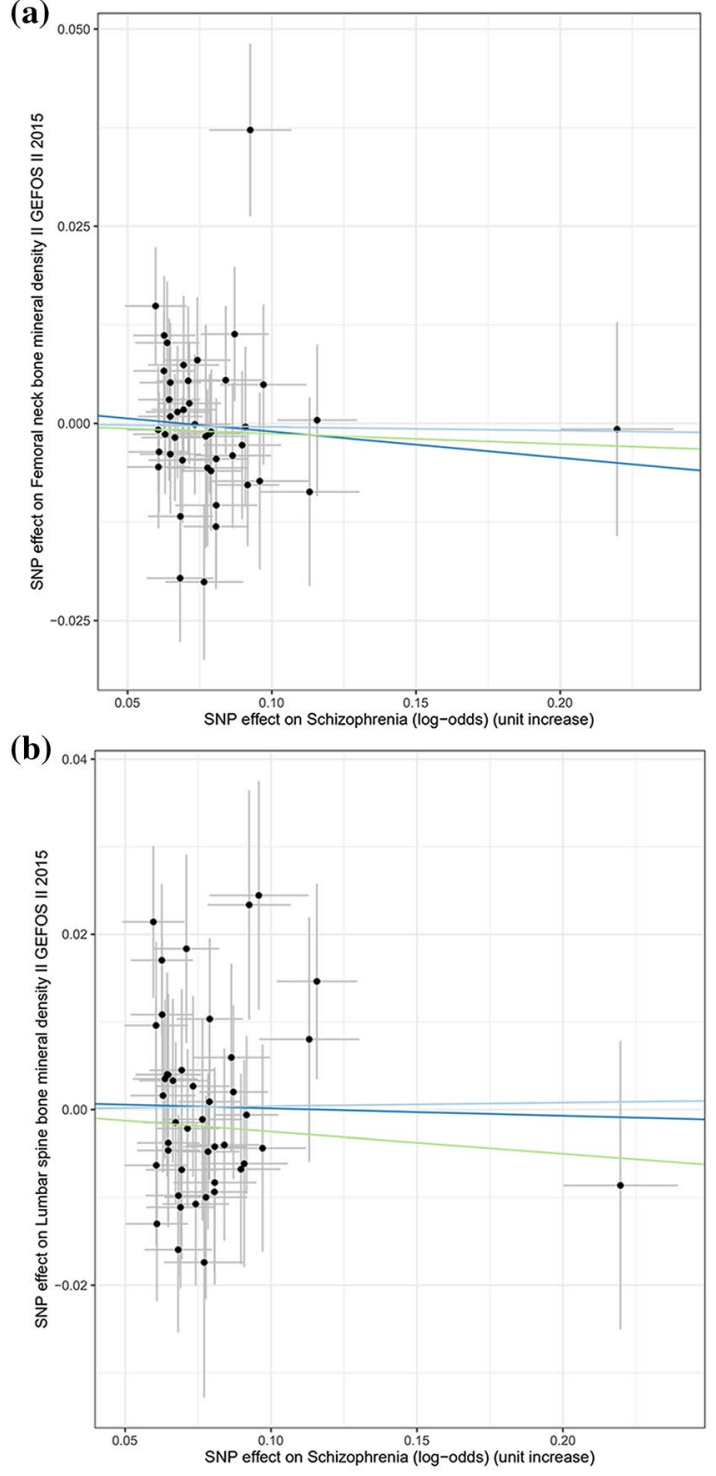

(c)

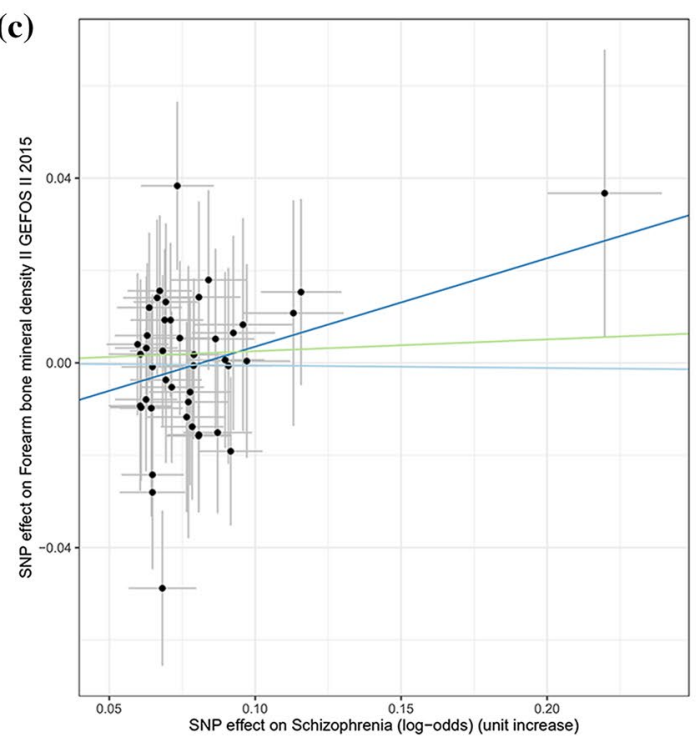

MR Test Methods

Inverse variance weighted

/ MR-Egger

Weighted median

Inverse variance weighted

/ MR-Egger

Weighted median

MR Test Methods

Inverse variance weighted

/ MR-Egger

Weighted median 


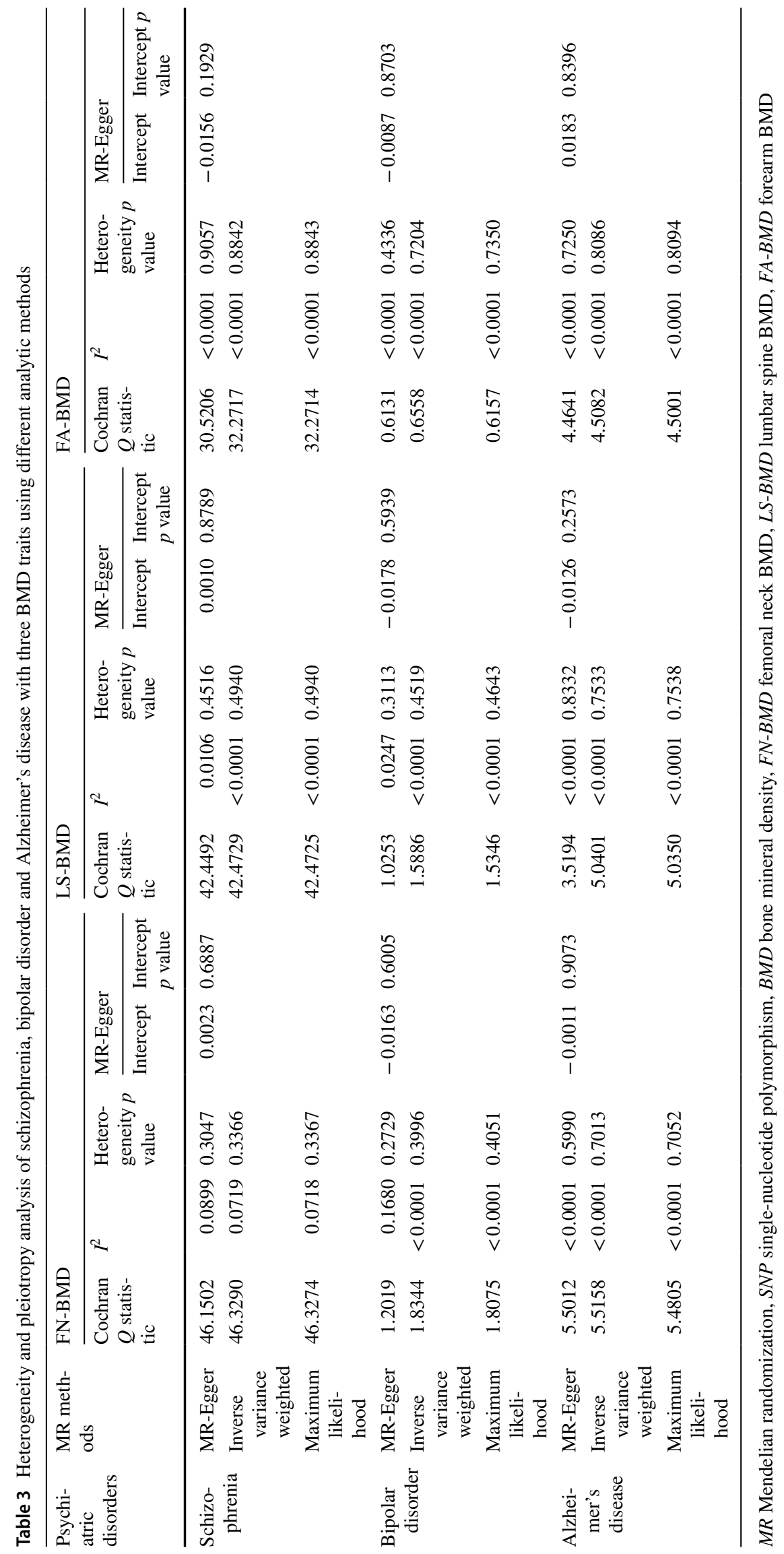




\section{Causality Between BD and BMD}

We chose three independent SNPs associated with BD in European ancestry [12] to perform the MR analysis for causality with FN-BMD, LS-BMD and FA-BMD, and no palindromic SNPs were found. None of the three SNPs were significantly associated with BMD outcomes $(p<0.0056)$ (e.g., 0.05/9) (Table 1). No evidence supported that the per unit increase in the log-odds of having the BD was causally associated with the FN-BMD and LS-BMD changes relied on IVW, WM and MR-Egger regression methods (For FN-BMD, MR-Egger OR 1.1355, $95 \%$ CI 0.8870 to $1.4537, p=0.4973$; IVW OR 1.0401 , $95 \%$ CI 0.9765 to $1.1079, p=0.2218$; WM OR 1.0552, $95 \%$ CI 0.9721 to $1.1453, p=0.1993$. For LS-BMD, MREgger OR $1.1819,95 \%$ CI 0.9073 to $1.5396, p=0.4323$; IVW OR $1.0738,95 \%$ CI 0.9978 to $1.1555, p=0.0572$; WM OR $1.0830,95 \%$ CI 0.9865 to $1.1890, p=0.0941$.) (Table 2, Fig. 2). However, the association between BD and FA-BMD was not consistent between three MR methods. The IVW method suggested a causal effect between $\mathrm{BD}$ and FA-BMD, while MR-Egger and WM suggested a null causal effect based on the Bonferroni-corrected significance threshold $(p<0.0167)$ (For FA-BMD, MR-Egger OR $1.2457,95 \%$ CI 0.7879 to $1.9693, p=0.5197$; IVW OR $1.1893,95 \%$ CI 1.0438 to $1.3552, p=0.0092$; WM OR $1.1829,95 \%$ CI 1.0058 to $1.3938, p=0.0448$.) (Table 2 , Fig. 2). Considering the improved power of causal effect detection and lower type I error of the WM method, we believed that MR results did not support the causal effect of the risk of BD with FA-BMD. We conducted the MREgger regression to assess the pleiotropy, and the results revealed that the pleiotropy was unlikely to bias the causality with FN-BMD (intercept $=-0.0163, p=0.6005$ ), LS-BMD (intercept $=-0.0178, p=0.5939$ ) and FA-BMD (intercept $=-0.0087, p=0.8703$ ) (Table 3). None of the three SNPs were significantly associated with known confounders at the Bonferroni-corrected significance threshold $(p<0.0167)$ (Tables S2-S7). We also found no significant heterogeneity between BD and BMD (For FN-BMD, MR-Egger $Q=1.2019, I^{2}=0.1680, p=0.2729$; IVW $Q=1.8344, I^{2}<0.0001, p=0.3996$; Maximum likelihood $Q=1.8075, I^{2}<0.0001, p=0.4051$. For LS-BMD, MR-Egger $Q=1.0253, I^{2}=0.0247, p=0.3113$; IVW $Q=1.5886, I^{2}<0.0001 p=0.4519$; Maximum likelihood $Q=1.5346, I^{2}<0.0001, p=0.4643$. For FA-BMD, MR-Egger $Q=0.6131, I^{2}<0.0001, p=0.4336$; IVW $Q=0.6558, I^{2}<0.0001, p=0.7204$; Maximum likelihood $Q=0.6157, I^{2}<0.0001, p=0.7350$.) (Table 3). The leaveone-out analysis and MR-Steiger directionality test showed that the precision and the direction of the IV estimates between higher risk of BD and BMD remained largely unchanged (Fig. S2, Table 2).

\section{Causality Between AD and BMD}

We obtained nine independent SNPs associated with AD in European ancestry [13] from summary statistics data sets of GWAS meta-analyses, and no palindromic SNPs were found. None of the nine SNPs were significantly associated with BMD outcomes $(p<0.0019)$ (e.g., 0.05/27) (Table 1). The increased risk of having AD was not significantly associated with the change of FN-BMD, LS-BMD and FA-BMD based on IVW, WM and MR-Egger regression methods (For FN-BMD, MR-Egger OR 1.0571, 95\% CI 0.9085 to $1.2299, p=0.4960$; IVW OR $1.0478,95 \%$ CI 0.9951 to $1.1034, p=0.0762$; WM OR $1.0438,95 \%$ CI 0.9756 to $1.1167, p=0.2139$. For LS-BMD, MR-Egger OR 1.1307, $95 \%$ CI 0.9509 to $1.3445, p=0.2070$; IVW OR 1.0207 , $95 \%$ CI 0.9620 to $1.0830, p=0.4982$; WM OR 1.0038 , 95\% CI 0.9286 to $1.0850, p=0.9248$. For FA-BMD, MREgger OR $0.9219,95 \%$ CI 0.6745 to $1.2601, p=0.6258$; IVW OR $0.9514,95 \%$ CI 0.8561 to $1.0574, p=0.3557$; WM OR $0.9334,95 \%$ CI 0.8147 to $1.0700, p=0.3225$.) (Table 2, Fig. 3). We found that the pleiotropy would not bias the causality with FN-BMD (intercept $=-0.0011, p=0.9073$ ), LS-BMD (intercept $=-0.0126, p=0.2573$ ) and FA-BMD (intercept $=0.0183, p=0.8396$ ) based on the MR-Egger regression (Table 3). We also checked the association of $\mathrm{AD}$ associated SNPs with confounders, and no association signal was detected among the nine SNPs we selected at the Bonferroni-corrected significance threshold $(p<0.05 / 9=0.0056)$ (Tables S2-S7). The heterogeneity test also demonstrated that there is no evidence of heterogeneity in our chosen SNPs (For FN-BMD, MR-Egger $Q=5.5012, I^{2}<0.0001$, $p=0.5990$; IVW $Q=5.5158, I^{2}<0.0001, p=0.7013$; Maximum likelihood $Q=5.4805, I^{2}<0.0001, p=0.7052$. For LSBMD, MR-Egger $Q=3.5194, I^{2}<0.0001, p=0.8332$; IVW $Q=5.0401, I^{2}<0.0001 p=0.7533$; Maximum likelihood $Q=5.0350, I^{2}<0.0001, p=0.7538$. For FA-BMD, MREgger $Q=4.4641, I^{2}<0.0001, p=0.7250$; IVW $Q=4.5082$, $I^{2}<0.0001, p=0.8086$; Maximum likelihood $Q=4.5001$, $I^{2}<0.0001, p=0.8094$ ) (Table 3). The leave-one-out analysis showed that none of the SNPs derived the majority of the association signal, and the MR-Steiger results supported the causal direction between AD and each BMD trait (Fig. S3, Table 2).

\section{Discussion}

There was evidence that risk factors contributing to reduction of BMD were associated with schizophrenia, BD and AD. For example, inflammatory factors play an important role in the progression of schizophrenia and BD $[25,26]$. Some inflammatory factors have been shown to have detrimental effects on BMD and increase the fracture risk 
Fig. 2 Scatter plots of genetic associations with BD against BMD using different MR methods. a BD and FN-BMD results; $\mathbf{b} \mathrm{BD}$ and LS-BMD results; and $\mathbf{c} \mathrm{BD}$ and $\mathrm{FA}-\mathrm{BMD}$ results. The slopes of each line represent the causal association for each method. The blue line represents the inverse variance weighted estimate, the green line represents the weighted median estimate, and the dark blue line represents the MREgger estimate
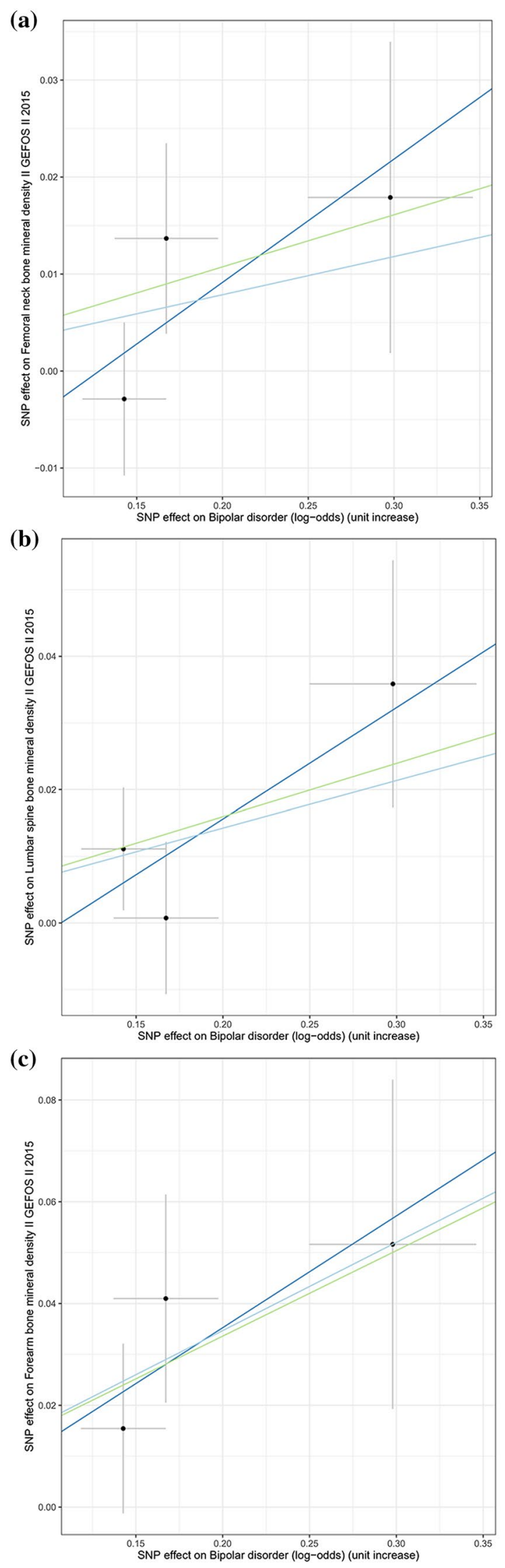

MR Test Methods

Inverse variance weighted

MR-Egger

Weighted median

MR Test Methods

Inverse variance weighted

/ MR-Egger

Weighted median

MR Test Methods

Inverse variance weighted

MR-Egger

Weighted median 
Fig. 3 Scatter plots of genetic associations with $\mathrm{AD}$ against BMD using different MR methods. a AD and FN-BMD results; $\mathbf{b} \mathrm{AD}$ and LS-BMD results; and $\mathbf{c} \mathrm{AD}$ and $\mathrm{FA}-\mathrm{BMD}$ results. The slopes of each line represent the causal association for each method. The blue line represents the inverse variance weighted estimate, the green line represents the weighted median estimate, and the dark blue line represents the MREgger estimate
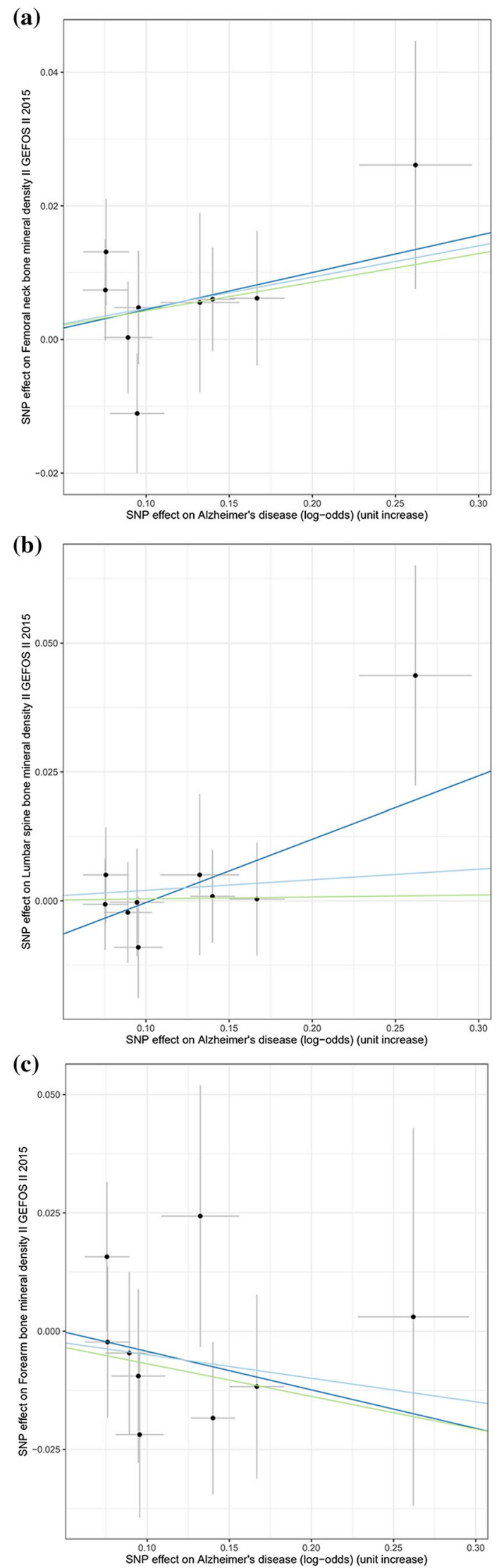

MR Test Methods

Inverse variance weighted

/ MR-Egger

Weighted median
MR Test Methods

Inverse variance weighted

/ MR-Egger

Weighted median
MR Test Methods

Inverse variance weighted

/ MR-Egger

Weighted median 
through the promotion of osteoclast differentiation and apoptosis in osteoblast populations [27]. Other risk factors of bone loss, such as age, sex, genetic susceptibility, estrogen and daily activity, were also associated with schizophrenia, $\mathrm{BD}$ and $\mathrm{AD}[3,27,28]$. However, it remained unclear whether these factors were causes or consequences of the three neuropsychiatric diseases. Therefore, the causal interfaces between the three neuropsychiatric diseases and BMD still remained unclear.

We selected SNPs with genome-wide association and independent inheritance without any LD as IVs to detect interactions between the three neuropsychiatric diseases and BMD at three measurement sites in the two-sample MR analysis. However, our MR analysis results did not help us distinguish statistical interactions between exposures and outcomes. To avoid the effects of confounding factors, we also detected the associations between the IVs and BMDrelated confounders (obesity indices (BMI, waist circumference, hip circumference and waist-to-hip circumference ratio), glycemic traits (type 2 diabetes, fasting glucose and 2-h glucose), systolic and diastolic blood pressure, smoking behavior, alcohol intake behavior and IBD). We performed heterogeneity and sensitivity tests and found low heterogeneity between SNPs and stable MR estimates, which indicated the reliability of the MR results. Furthermore, we used the MR-Egger method to detect and adjust for pleiotropy of the genetic variants. It was revealed that directional pleiotropy was unlikely to bias the MR results. In summary, our results did not provide evidence to support the causal effects of the increased risk of the three neuropsychiatric diseases (schizophrenia, $\mathrm{BD}$ and $\mathrm{AD}$ ) with $\mathrm{BMD}$ at three measurement sites (FN, LS and FA).

To the best of our knowledge, this is the first MR analysis to investigate the causal association between three neuropsychiatric diseases (schizophrenia, BD and AD) and BMD. The results of our novel study were inconsistent with most observational studies. Schizophrenia is one of the most severe psychiatric disorders in the world and can lead to many complications and disability [3]. Osteoporosis and osteopenia are common diseases associated with schizophrenia. Although some studies revealed no significant differences in BMD between patients with schizophrenia and healthy controls [29], most studies reported a higher prevalence of osteoporosis and osteopenia in patients with schizophrenia, which has attracted clinical attention $[6,30$, 31]. Cui et al. [6] carried out a cross-sectional study and suggested a higher prevalence of osteoporosis and low-bone mass in Chinese schizophrenia patients by measuring BMD at the heel. Stubbs et al. [30] executed one meta-analysis in Western countries and showed that patients with schizophrenia are almost twice as likely as age- and sex-matched controls to have low-bone mass. One large populationcohort study also found that patients with schizophrenia are at a significantly higher risk of hip fractures compared to the general population [31]. It is worth discussing the reasons why the reduction of BMD occurred in schizophrenia patients. As previously reported, there are many risk factors in schizophrenia patients that affect BMD. For example, it was reported that patients with schizophrenia typically have reduced levels of physical activity [32] and high levels of smoking [33]. Antipsychotic drug intake might also have potential negative effects on the bone quality. Howard et al. [34] found that prolactin-raising antipsychotic medication was associated with the prevalence of hip fracture in patients with a history of schizophrenia. As a result of many confounding factors associated with BMD, it was very difficult to identify the causal association between schizophrenia and BMD by traditional epidemiological studies. We performed the MR analysis to mitigate the confounding factors' effects and offered suggestive evidence that there was no significant causal association between schizophrenia and BMD value, despite differences from previous epidemiological findings.

BD refers to a group of affective disorders in psychiatry, which is characterized by multiple depressive or manic episodes [35]. Recent data have revealed increased fracture risk in patients with BD [7, 28, 36]. Hsu et al. [7] conducted a retrospective population-based cohort study and found that patients with BD have a higher risk of subsequent fracture. They demonstrated that BD was an independent risk factor for fracture. Su et al. [36] reported that bipolar patients had a higher risk of fracture compared to matched controls. However, there have been few epidemiological studies on BMD changes in bipolar patients because of many confounding factors between BD and BMD. It was hypothesized that abnormal inflammatory actions, mitochondrial dysfunction, oxidative stress and endocrine factors in BD [4] might damage bone quality. Some studies also reported that the drugs in the treatment of $\mathrm{BD}$, such as anticonvulsants and antipsychotics $[34,37]$, would be independently harmful to bone. Shen et al. [37] conducted a meta-analysis and found a robust association between the use of anticonvulsants and fracture risk. The behavioral manifestations of BD might also increase the risk of fracture. Some bipolar patients were impulsive, hyperactive and violent. These behaviors exposed patients to the dangers of physical injury and fracture [38]. Although many studies revealed the possible association between $\mathrm{BD}$ and $\mathrm{BMD}$, our MR analysis results did not support that the association between increased risk of $\mathrm{BD}$ and BMD change is consistent with a causal effect.

$\mathrm{AD}$ is the commonest cause of dementia and is characterized by age-related degenerative disorders [5]. There were too many common risk factors associated with $\mathrm{AD}$ and BMD, such as BMI, smoking behavior and alcohol intake [39], so it was difficult to explore the causal association between them. However, it was particularly important to identify the causality because fracture and 
fracture-associated complications can exacerbate the healthcare burden and increase early mortality in patients with AD [40]. It was revealed that patients with $\mathrm{AD}$ had a 3.2 times greater risk of hip fracture than non-AD patients after controlling for confounding factors [8]. However, patients with $\mathrm{AD}$ often have problems with gait as the disease progresses [41], which might increase the propensity to fall down and increase the risk of hip fracture. Some investigators [42] believe that sarcopenia and decreased mobility, which are often associated with AD, detrimentally impacted the bone quality. Data from other studies indicate that weight loss often occurs in patients with $\mathrm{AD}$, which might contribute to the bone loss in AD [43]. Some studies suggest that lower BMD is associated with the higher risk of AD in Western population [44]. Zhou et al. [45] also report that low BMD could predict the likelihood of progression to $\mathrm{AD}$ in patients with mild cognitive impairment. Although there are complicated associations between $\mathrm{AD}$ and $\mathrm{BMD}$, we performed a MR analysis and found that there was no significant causal associations of AD with BMD.

This MR analysis has several important strengths to note $[9,10]$. First, the causal effects of three neuropsychiatric diseases with BMD value were not distorted by residual confounding factors, which were mitigated through the use of genetic variants as proxies. Second, we performed sensitivity analysis and found that the final causal effect estimates were relatively consistent. Then, to eliminate pleiotropic effects, we used MR-Egger regression and found no evidence that pleiotropy affects the results. Moreover, it was confirmed that the schizophrenia, BD and AD-associated genetic variants were not significantly associated with other phenotypes with a potential or established association with BMD. Therefore, we are able to determine the causal associations with high precision. The present study has several limitations. First, although the causal associations between three neuropsychiatric diseases and BMD were discussed in our study, we did not analyze the causal associations between three neuropsychiatric diseases and fractures. Fractures and their complications are severe and common clinical problems for the patients with schizophrenia, BD or AD [6-8], so it is necessary to take the causal associations into account in the further research. Second, the associations of the SNPs with schizophrenia and BMD were assessed in different subgroups of the population. The GWAS study of schizophrenia was performed in European and Asian ancestry, while the study of BMD was performed in European ancestry. The SNPs related to schizophrenia as IVs could potentially be biased by population stratification if the frequency of SNPS differs widely across populations [10]. Third, our analysis included only three SNPs as IVs on BD and might have had limited power to detect an association between BD and BMD. We could increase the statistical power and obtain a more precise result by combining multiple genetic variants together [10]. Fourth, we only evaluated the associations between SNPs and key potential confounders due to the limited publicly available GWAS databases. The associations between these instruments and other potential confounders such as exercise and rheumatoid arthritis, were not evaluated in our study. The sample size was another possible limitation. Although our study used a large sample size from GWAS in the MR Base database, which enabled us to estimate the causal effects more precisely than using individuallevel data from small studies, the sample size might still be too low to detect the effect of the increased risk of having schizophrenia, BD and AD with BMD changes.

\section{Conclusion}

In summary, this MR analysis did not suggest the significant causal effects of the increased risk of having schizophrenia, bipolar disorder or Alzheimer's disease with bone mineral density at the femoral neck, lumbar spine and forearm. The complicated links between the three neuropsychiatric diseases with BMD might be influenced by other confounding factors, which still need further investigation in the future. In addition, well-designed epidemiological and MR studies using more IVs can help to further confirm or rule out causality between the three neuropsychiatric diseases and BMD.

Acknowledgements This work was supported by grants from Beijing Municipal Science and Technology Commission (Z181100001718195). The authors also wish to thank Professor Yueqin Huang in Peking University Sixth Hospital for her advice in preparation of this manuscript. The authors also thank the DSC (Wuhan) Scientific Co.Ltd. for consulting services on data management, statistical analysis, and scientific writing.

Author Contributions YT and ZC conceptualized and designed the study. XM and SZ provided the "TwoSampleMR" package codes in $\mathrm{R}$ language and analyzed the data in the study. ZC and SZ drafted the manuscript. ZL and FZ gave constructive suggestions when writing the manuscript. All the authors have read the manuscript.

\section{Compliance with Ethical Standards}

Conflict of interest Zhiyong Cui, Xiangyu Meng, Siying Zhuang, Zhaorui Liu, Fang Zhou and Yun Tian declared that there were no conflicts of interest to report.

Human and Animal Rights and Informed Consent This article does not contain any studies with human participants or animals performed by any of the authors.

Open Access This article is distributed under the terms of the Creative Commons Attribution 4.0 International License (http://creativeco mmons.org/licenses/by/4.0/), which permits unrestricted use, distribution, and reproduction in any medium, provided you give appropriate credit to the original author(s) and the source, provide a link to the Creative Commons license, and indicate if changes were made. 


\section{References}

1. Lorentzon M, Cummings SR (2015) Osteoporosis: the evolution of a diagnosis. J Intern Med 277:650-661

2. Kanis JA, Melton LR 3rd, Christiansen C, Johnston CC, Khaltaev N (1994) The diagnosis of osteoporosis. J Bone Miner Res 9:1137-1141

3. Owen MJ, Sawa A, Mortensen PB (2016) Schizophrenia. Lancet 388:86-97

4. Vieta E, Berk M, Schulze TG, Carvalho AF, Suppes T, Calabrese JR et al (2018) Bipolar disorders. Nat Rev Dis Primers 4:18008

5. Lane CA, Hardy J, Schott JM (2018) Alzheimer's disease. Eur J Neurol 25:59-70

6. Cui J, Liu H, Shao J, Xu DM, Wang Y, Fei Z et al (2018) Prevalence, risk factors and clinical characteristics of osteoporosis in Chinese inpatients with schizophrenia. Schizophr Res 195:488-494

7. Hsu CC, Hsu YC, Chang KH, Lee CY, Chong LW, Wang YC et al (2016) Increased risk of fracture in patients with bipolar disorder: a nationwide cohort study. Soc Psychiatry Psychiatr Epidemiol 51:1331-1338

8. Baker NL, Cook MN, Arrighi HM, Bullock R (2011) Hip fracture risk and subsequent mortality among Alzheimer's disease patients in the United Kingdom, 1988-2007. Age Ageing 40:49-54

9. Emdin CA, Khera AV, Kathiresan S (2017) Mendelian randomization. JAMA 318:1925-1926

10. Larsson SC, Michaëlsson K, Burgess S (2018) Mendelian randomization in the bone field. Bone 126:51-58

11. Ripke S, Neale BM, Corvin A, Walters JT, Farh KH, Holmans PA et al (2014) Biological insights from 108 schizophrenia-associated genetic loci. Nature 511:421-427

12. Sklar P, Ripke S, Scott LJ, Andreassen OA, Cichon S, Craddock $\mathrm{N}$ et al (2011) Large-scale genome-wide association analysis of bipolar disorder identifies a new susceptibility locus near ODZ4. Nat Genet 43:977-983

13. Lambert JC, Ibrahim-Verbaas CA, Harold D, Naj AC, Sims R, Bellenguez C et al (2013) Meta-analysis of 74,046 individuals identifies 11 new susceptibility loci for Alzheimer's disease. Nat Genet 45:1452-1458

14. Zheng HF, Forgetta V, Hsu YH, Estrada K, Rosello-Diez A, Leo $\mathrm{PJ}$ et al (2015) Whole-genome sequencing identifies EN1 as a determinant of bone density and fracture. Nature 526:112-117

15. Locke AE, Kahali B, Berndt SI, Justice AE, Pers TH, Day FR et al (2015) Genetic studies of body mass index yield new insights for obesity biology. Nature 518:197-206

16. Randall JC, Winkler TW, Kutalik Z, Berndt SI, Jackson AU, Monda KL et al (2013) Sex-stratified Genome-wide association studies including 270,000 individuals show sexual dimorphism in genetic loci for anthropometric traits. PLoS Genet 9:e1003500

17. Shungin D, Winkler TW, Croteau-Chonka DC, Ferreira T, Locke AE, Mägi R et al (2015) New genetic loci link adipose and insulin biology to body fat distribution. Nature 518:187-196

18. Morris AP, Voight BF, Teslovich TM, Ferreira T, Segrè AV, Steinthorsdottir V et al (2012) Large-scale association analysis provides insights into the genetic architecture and pathophysiology of type 2 diabetes. Nat Genet 44:981-990

19. Dupuis J, Langenberg C, Prokopenko I, Saxena R, Soranzo N, Jackson AU et al (2010) New genetic loci implicated in fasting glucose homeostasis and their impact on type 2 diabetes risk. Nat Genet 42:105-116

20. Saxena R, Hivert MF, Langenberg C, Tanaka T, Pankow JS, Vollenweider $P$ et al (2010) Genetic variation in GIPR influences the glucose and insulin responses to an oral glucose challenge. Nat Genet 42:142-148
21. Ehret GB, Munroe PB, Rice KM, Bochud M, Johnson AD, Chasman DI et al (2011) Genetic variants in novel pathways influence blood pressure and cardiovascular disease risk. Nature 478:103-109

22. Furberg H, Kim Y, Dackor J, Boerwinkle E, Franceschini N, Ardissino D et al (2010) Genome-wide meta-analyses identify multiple loci associated with smoking behavior. Nat Genet 42:441-447

23. Clarke TK, Adams MJ, Davies G, Howard DM, Hall LS, Padmanabhan S et al (2017) Genome-wide association study of alcohol consumption and genetic overlap with other healthrelated traits in UK Biobank $(\mathrm{N}=112,117)$. Mol Psychiatry 22:1376-1384

24. Liu JZ, van Sommeren S, Huang H, Ng SC, Alberts R, Takahashi A et al (2015) Association analyses identify 38 susceptibility loci for inflammatory bowel disease and highlight shared genetic risk across populations. Nat Genet 47:979-986

25. Khandaker GM, Cousins L, Deakin J, Lennox BR, Yolken R, Jones PB (2015) Inflammation and immunity in schizophrenia: implications for pathophysiology and treatment. Lancet Psychiatry 2:258-270

26. Tanaka T, Matsuda T, Hayes LN, Yang S, Rodriguez K, Severance EG et al (2017) Infection and inflammation in schizophrenia and bipolar disorder. Neurosci Res 115:59-63

27. Pouresmaeili F, Kamali Dehghan B, Kamarehei M, Goh YM (2018) A comprehensive overview on osteoporosis and its risk factors. Ther Clin Risk Manag 14:2029-2049

28. Chandrasekaran V, Brennan-Olsen SL, Stuart AL, Pasco JA, Berk M, Hodge JM et al (2019) Bipolar disorder and bone health: A systematic review. J Affect Disord 249:262-269

29. Doknic M, Maric NP, Britvic D, Pekic S, Damjanovic A, Milijic $\mathrm{D}$ et al (2011) Bone remodeling, bone mass and weight gain in patients with stabilized schizophrenia in real-life conditions treated with long-acting injectable risperidone. Neuroendocrinology 94:246-254

30. Stubbs B, De Hert M, Sepehry AA, Correll CU, Mitchell AJ, Soundy A et al (2014) A meta-analysis of prevalence estimates and moderators of low bone mass in people with schizophrenia. Acta Psychiatr Scand 130:470-486

31. Sørensen H, Jensen SO, Nielsen J (2013) Schizophrenia, antipsychotics and risk of hip fracture: a population-based analysis. Eur Neuropsychopharmacol 23:872-878

32. Vancampfort D, De Hert M, De Herdt A, Soundy A, Stubbs B, Bernard P et al (2014) Associations between perceived neighbourhood environmental attributes and self-reported sitting time in patients with schizophrenia: a pilot study. Psychiatry Res 215:33-38

33. Freeman TP, Stone JM, Orgaz B, Noronha LA, Minchin SL, Curran HV (2013) Tobacco smoking in schizophrenia: investigating the role of incentive salience. Psychol Med 44:2189-2197

34. Howard L, Kirkwood G, Leese M (2007) Risk of hip fracture in patients with a history of schizophrenia. Br J Psychiatry 190:129-134

35. Phillips ML, Kupfer DJ (2013) Bipolar disorder diagnosis: challenges and future directions. Lancet 381:1663-1671

36. Su JA, Cheng BH, Huang YC, Lee CP, Yang YH, Lu ML et al (2017) Bipolar disorder and the risk of fracture: a nationwide population-based cohort study. J Affect Disord 218:246-252

37. Shen C, Chen F, Zhang Y, Guo Y, Ding M (2014) Association between use of antiepileptic drugs and fracture risk: a systematic review and meta-analysis. Bone 64:246-253

38. Mason L, O'Sullivan N, Montaldi D, Bentall RP, El-Deredy W (2014) Decision-making and trait impulsivity in bipolar disorder are associated with reduced prefrontal regulation of striatal reward valuation. Brain 137:2346-2355 
39. Zhou R, Deng J, Zhang M, Zhou HD, Wang Y (2011) Association between bone mineral density and the risk of Alzheimer's disease. J Alzheimers Dis 24:101-108

40. Serrano-Aguilar PG, Lopez-Bastida J, Yanes-Lopez V (2006) Impact on health-related quality of life and perceived burden of informal caregivers of individuals with Alzheimer's disease. Neuroepidemiology 27:136-142

41. Gras LZ, Kanaan SF, McDowd JM, Colgrove YM, Burns J, Pohl PS (2015) Balance and gait of adults with very mild Alzheimer disease. J Geriatr Phys Ther 38:1-7

42. Ogawa Y, Kaneko Y, Sato T, Shimizu S, Kanetaka H, Hanyu H (2018) Sarcopenia and muscle functions at various stages of Alzheimer disease. Front Neurol 9:710

43. Buchman AS, Wilson RS, Bienias JL, Shah RC, Evans DA, Bennett DA (2005) Change in body mass index and risk of incident Alzheimer disease. Neurology 65:892-897
44. Tan ZS, Seshadri S, Beiser A, Zhang Y, Felson D, Hannan MT et al (2005) Bone mineral density and the risk of Alzheimer disease. JAMA Neurol 62:107-111

45. Zhou R, Zhou H, Rui L, Xu J (2014) Bone loss and osteoporosis are associated with conversion from mild cognitive impairment to Alzheimer's disease. Curr Alzheimer Res 11:706-713

Publisher's Note Springer Nature remains neutral with regard to jurisdictional claims in published maps and institutional affiliations. 